\title{
SELEÇÃO DE GENÓTIPOS DE BATATA-DOCE Ipomoea batatas (L.) Lam. COM CARACTERÍSTICAS PARA CONSUMO DE MESA.
}

Selection of sweet potato genotypes Ipomoea batatas (L.) Lam. with characteristics for table consumption.

Selección de genótipos de patata dulce Ipomoea batatas (L.) Lam. com características para el consumo de la mesa.

Rosinete Nogueira de Sousa*1, Illys Janes Alves de Sousa, Wesley Rosa de Santana ${ }^{3}$, Mateus Rodrigues Brito ${ }^{4}$, Flavia Lucila Tonani Siqueira ${ }^{5}$.

${ }^{1}$ Mestranda em Agroenergia, Universidade Federal do Tocantins, Palmas, Brasil.

${ }^{2}$ Mestre em Agroenergia, Universidade Federal do Tocantins, Palmas, Brasil.

${ }^{3}$ Laboratório de Sistema de Produção de Energia a Partir de Fontes Renováveis (LASPER), Universidade Federal do Tocantins, Palmas, Brasil.

${ }^{4}$ Mestrando em Ciências do Ambiente, Universidade Federal do Tocantins, Palmas, Brasil.

${ }^{5}$ Docente do curso de Engenharia Ambiental, Universidade Federal do Tocantins, Palmas, Brasil.

*Correspondência: Universidade Federal do Tocantins, Av. NS 15, 109 Norte, Palmas, Tocantins, Brasil. CEP:77.010-090.e-mail ns.rosinete@gmail.com

Artigo recebido em 28/08/2018 aprovado em 20/03/2020 publicado em 27/03/2020.

\section{RESUMO}

A batata-doce é um alimento, que possui uma grande variabilidade genética, podendo ser selecionadas para algumas finalidades como, por exemplo: maior resistência a pragas e doenças. Pesquisadores da Universidade Federal do Tocantins -UFT- vem realizando desde 1997, estudos com batata-doce, destinada a produção de etanol e desde, então, vem sendo realizados melhoramento genético entre genótipos com o intuito de melhorar a produção do biocombustível. Entretanto, existem genótipos com características desejáveis ao consumo humano. Diante disso, o objetivo deste trabalho foi avaliar alguns desses genótipos, com intuito de encontrar características adequadas para mesa. As análises realizadas foram: formato, resistência a insetos, cor de casca e cor de polpa. Foram avaliadas 30 cultivares, porém, duas morreram. As cultivares HSF 0506 e BDI 2011-52 apresentaram bom formato de raiz (nota 1). As cultivares BDI 2011-52 (nota 1,11) e HSF 0506 (nota 1,33) se destacaram com melhores notas em resistência a insetos. A cor predominante de casca e polpa foi branca (64\%) e creme (71\%), respectivamente. Segundo os dados avaliados, a cultivar HSF 0506 se mostrou superior às outras, atendendo os padrões de mesa solicitados pelo consumidor.

Palavras-chave: Padrões de mesa da batata-doce; Melhoramento genético.

\section{ABSTRACT}

Sweet potato is a food that has a great genetic variability, in which it can be selected for some purposes such as: greater resistance to pests and diseases. Researchers at the Federal University of Tocantins (UFT) have been conducting studies with sweet potatoes since 1997 to produce ethanol. Since then, genetic improvement between genotypes has been carried out in order to improve biofuel production. However, there are genotypes with characteristics desirable for human consumption. Therefore, the objective of this work was to evaluate some of these genotypes, in order to find suitable table characteristics. The analyzes were: size, insect resistance, bark color and pulp color. Thirty cultivars were evaluated, in which two died. The cultivars HSF 0506 and BDI 2011-52 presented good root format (note 1). The cultivars BDI $2011-52$ (note 1.11) and HSF 0506 (note 1.33) stood out with better 
notes on insect resistance. The predominant color of bark and pulp was white (64\%) and cream (71\%), respectively. According to the data evaluated, the cultivar HSF 0506 was superior to the others, taking into account the table standards requested by the consumer.

Key words: Sweet potato table patterns; Genetical enhancement.

\section{RESUMEN}

La patata dulce es un alimento, que posee una gran variabilidad genética, en el cual pueden ser seleccionadas para algunas finalidades como, por ejemplo: mayor resistencia a plagas y enfermedades. Investigadores de la Universidad Federal de Tocantins -UFT- vienen realizando desde 1997, estudios con patata dulce, destinada a la producción de etanol. Desde entonces se está realizando un mejoramiento genético entre genotipos con el fin de mejorar la producción del biocombustible. Sin embargo, existen genotipos con características deseables al consumo humano. Por ello, el objetivo de este trabajo fue evaluar algunos de estos genotipos, con el propósito de encontrar características adecuadas para la mesa. Los análisis realizados fueron: formato, resistencia a insectos, color de cáscara y color de pulpa. Se evaluaron 30 cultivares, en los que dos murieron. Los cultivares HSF 0506 y BDI 2011 52 presentaron buen formato de raíz (nota 1). Los cultivares BDI 2011 -52 (nota 1,11) y HSF 0506 (nota 1,33) se destacaron con mejores notas en resistencia a insectos. El color predominante de cáscara y pulpa fue blanco (64\%) y crema (71\%), respectivamente. Según los datos evaluados, El cultivar HSF 0506 se mostró superior a las otras, atendiendo a los patrones de mesa solicitados por el consumidor.

Palabras clave: Patrones de mesa de la patata dulce; Mejoramiento genético.

\section{INTRODUÇÃO}

A batata-doce (Ipomoea batatas) é uma hortaliça dicotiledônea pertencente á família das Convolvulacae (SILVA et al., 2008). É utilizada para a alimentação humana, animal e recentemente na produção de etanol. (CAMARGO et al., 2016).

O uso na alimentação é atribuído ao fato de ser uma rica fonte de minerais e vitamina, além de possuir baixo custo. De acordo com Furlaneto et al. (2012) a mesma é rica em carboidratos, variando de 13 a 29\%, vitamina $\mathrm{C}$ e complexo B.

É uma cultura rústica e de boa adaptação a solos de baixa fertilidade e condições climáticas, favorecendo dessa forma, seu o cultivo. (SILVA et al., 2019). O clima é um dos principais fatores para o desenvolvimento satisfatório da planta. A cultura exige temperaturas mais elevadas (acima de $24^{\circ} \mathrm{C}$ ) (SILVA et al., 2008; NEUMANN et al., 2017).

Existem diversas variedades dessa cultura. Estas podem ser classificadas levando em consideração as seguintes variáveis: cor interna e externa, tamanho, formato, cor de folhas e flores (MIRASSE, 2010; FURLANETO et al., 2012).

$\mathrm{O}$ fato de possuir uma alta variabilidade genética favorece as possibilidades de aprimoramento da cultura, como: maior resistência a pragas e doenças, teor de matéria seca, tamanho e produtividade (SANTANA, 2013; BEVILAQUA et al., 2019).

Kalkmann (2011) diz que:

"A exploração de todo o potencial de clones
de batata-doce envolve trabalhos de pesquisa
específica nas áreas de melhoramento
genético, com avaliação de rendimento,
qualidade raiz, resistência energética,
adaptações a diversas regiões e que possuam
as mais diversas aptidões, entre elas
alimentação humana e animal."

Pesquisadores da Universidade Federal do Tocantins -UFT- vem realizando desde 1997, estudos com batata-doce, destinada a produção de etanol. Desde então vem sendo realizados melhoramento genéticos entre genótipos de batata-doce industrial com o intuito de aprimorar esta cultura para a produção do 
biocombustível. Muitos desses genótipos melhorados podem apresentar aptidões para consumo de mesa.

Diante disto, o presente trabalho teve por finalidade avaliar genótipos de batata-doce industrial pertencentes ao banco de germoplasma do Laboratório de Produção de Energia a Partir de Fontes Renováveis (LASPER/UFT), a fim de verificar se há características atrativas para o consumo humano.

\section{MATERIAIS E MÉTODOS}

\section{Caracterização da área experimental}

A área utilizada para a realização do experimento foi o Centro Tecnológico Agroindustrial e Ambiental - CTAA, do Campus Universitário de Palmas, Universidade Federal do Tocantins - UFT situado nas coordenadas S $10^{\circ} 10^{\prime} 42,1$, W $48^{\circ} 21^{\text {' }}$ 22,6", altitude de 216.

O solo da área em estudo é do grupo Latossolos, que são caracterizados como profundos e bem drenados. O clima característico é o tropical, com verão úmido e inverno com período de estiagem com temperaturas anual média de $27^{\circ} \mathrm{C}$ e precipitação média anual de 1700-1800 mm (SEPLAN, 2012).

\section{Caracterização do experimento}

O delineamento experimental utilizado foi inteiramente casualizado, representado por trinta tratamentos (genótipos) com três repetições, contendo 6 plantas em cada parcela, totalizando 540 plantas. Os tratamentos constituíram-se de genótipos provenientes do banco de germoplasma do laboratório LASPER/UFT, sendo este pertencente ao programa de melhoramento genético para a cultura da batata-doce.

Utilizou-se como testemunha a cultivar de batata-doce: Brazlândia roxa, desenvolvida pela Embrapa, por apresentar bons aspectos comerciais para consumo de mesa como: polpa creme, tamanho médio e formato alongado. A mesma possui película externa roxa, polpa creme, doce, que após o cozimento tornase creme-amarelada e é bem seca, com baixo teor de fibras. É uma cultivar tardia, devendo ser colhida após 150 dias. Apresenta, também, boa resistência à pragas de solo. A produtividade média obtida na Embrapa Hortaliças foi de $25 \mathrm{t} / \mathrm{ha}$, em ciclos de cinco meses.

O experimento iniciou com a produção de mudas dos genótipos em canteiros previamente preparados e adubados, onde permaneceram por mais de 60 dias.

O preparo da área experimental ocorreu por meio de uma aração e gradagem. O plantio foi realizado através das ramas dos genótipos do banco de germoplasma do LASPER. As ramas foram alocadas em leiras com espaçamentos de $0,90 \mathrm{~m}$ entre leiras e $0,35 \mathrm{~m}$ entre plantas.

Os tratos culturais e adubação foram efetuados sempre que necessários conforme recomendação da cultura da batata-doce proposto por Miranda et al. (1995). Após a colheita, as batatas-doces foram armazenadas separadamente e posteriormente avaliadas, considerando as seguintes variáveis:

a. Formato: através de uma escala de nota, que variam de 1 a 5 (quadro 1) estabelecida por França et.al. (1983) citado por Azevedo et al. (2000).

b. Resistência a insetos de solo: determinada, assim como na avaliação do formato, através de uma escala de notas, que varia de 1 a 5 (quadro 2) estabelecida por França et.al. (1983), citado por Azevedo et al. (2000).

c. Cor de casca e polpa.

As notas foram dadas por três avaliadores, sendo o valor final expresso pela média dos três. 
Quadro 1. Escala notas atribuídas ao formato da batata-doce.

\begin{tabular}{|c|l|}
\hline NOTAS & \multicolumn{1}{c|}{ CLASSIFICAÇÃO } \\
\hline 1 & Raízes com formato fusiforme, regular, sem veias ou qualquer rachadura. \\
\hline 2 & Raízes com formato considerado bom, próximo de fusiforme, com algumas veias. \\
\hline 3 & Raízes com formato desuniforme, com veias e bastante irregular. \\
\hline 4 & Raízes muito grandes, com veias e rachaduras (indesejável comercialmente). \\
\hline 5 & Raízes totalmente fora dos padrões comerciais, muito irregulares e com muitas veias e rachaduras. \\
\hline
\end{tabular}

Fonte: Adaptado por Azevedo et al., 2000.

Quadro 2. Escala de notas atribuídas a danos causados por insetos de solo em genótipos de batata-

\begin{tabular}{|c|l|}
\hline NOTAS & \multicolumn{1}{c|}{ CLASSIFICAÇÃO } \\
\hline 1 & Raízes livres de danos, com aspecto comercial desejável. \\
\hline 2 & $\begin{array}{l}\text { Raízes com poucos danos, perdendo um pouco com relação aos aspectos comercial (presença de algumas } \\
\text { galerias e furos nas raízes). }\end{array}$ \\
\hline 3 & $\begin{array}{l}\text { Raízes com danos verificados sem muito esforço virtual (presença de galerias e furos nas raízes em maior } \\
\text { intensidade), com aspectos comerciais prejudicados. }\end{array}$ \\
\hline 4 & $\begin{array}{l}\text { Raízes com muitos danos, praticamente imprestáveis para comercialização (presença de muitas galerias, furos } \\
\text { e início de apodrecimento). }\end{array}$ \\
\hline 5 & $\begin{array}{l}\text { Raízes totalmente imprestáveis para fins comerciais (repletas de galerias, furos e apodrecimento mais } \\
\text { avançado). }\end{array}$ \\
\hline
\end{tabular}

Fonte: Adaptado por Azevedo et al., 2000.

\section{RESULTADOS E DISCUSSÃO}

De acordo com os dados da tabela 1, percebese que das 30 cultivares plantadas 28 sobreviveram. Para a avaliação de formato, nota-se que as cultivares HSF 0506 e BDI 2011-52 apresentaram bom formato de raiz (nota 1), enquanto que a BDI 2011-67 apresentou pior formato entra as cultivares (nota 5).

As cultivares BDI 2011-52 (nota 1,11) e HSF 0506 (nota 1,33), também se destacaram com melhores notas quanto à resistência a insetos. Já a BDI 2011-67 mostrou-se com menor resistência a insetos (nota 3,67).

A cultivar testemunha, Brazlândia Roxa, apresentou-se com boas características de formato (nota 1,44$)$ e resistência à insetos (nota 1,67$)$, além de possuir cor de casca roxa e polpa creme, aspectos estes mais procurados pelos consumidores.
As figuras 1 e 2 mostra a quantidade em porcentagem (\%) de cor de casca e polpa, respectivamente, das cultivares avaliadas.

Figura 1. Percentual de cor de casca das batatas-doces. Cor de casca (\%)

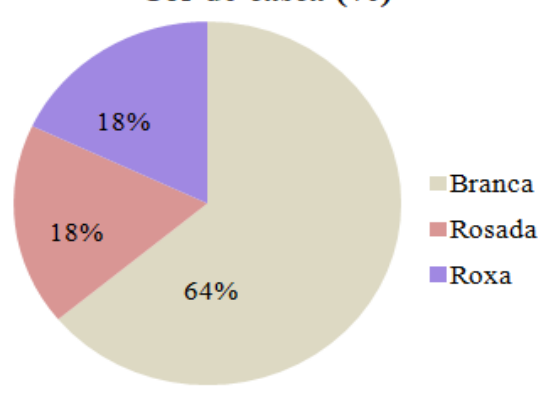

Figura 2. Percentual de cor de polpa das batatas-doces. 
Tabela 1. Médias para formato de raiz, danos causados por insetos de solo e colorações de casca e polpa em 28 clones de batata-doce.

\begin{tabular}{|c|c|c|}
\hline Genótipos & Formato & $\overline{\text { Resistência a insetos }}$ \\
\hline 2007 HSF 8214 & 2,78 & 3,56 \\
\hline BDI 2011 3C & 3,78 & 2,22 \\
\hline 2007 HSF 0121 & 2,11 & 2,67 \\
\hline PA 36 & 2,22 & 1,56 \\
\hline 2007 HSF 1105 & 2,00 & 2,33 \\
\hline BDI 2011-78 & 3,00 & 3,22 \\
\hline BDI 2011-81 & 1,89 & 2,11 \\
\hline BDI 2011-72 & 3,56 & 2,56 \\
\hline BDI 2007-56 & 1,67 & 2,33 \\
\hline HSF 2816 & 1,44 & 2,44 \\
\hline BD 165 & 1,56 & 2,78 \\
\hline BDI 2011-67 & 5,00 & 3,67 \\
\hline BDI 2011-94 & 3,00 & 3,00 \\
\hline PA-18 & 1,67 & 2,22 \\
\hline BDI 2011-52 & 1,00 & 1,11 \\
\hline BDI 155 & 3,33 & 3,22 \\
\hline $26 \mathrm{BD}(\mathrm{BAxBE})$ & 2,22 & 2,33 \\
\hline 2007 HSF-0417 & 1,89 & 1,89 \\
\hline BD 150 & 2,67 & 1,89 \\
\hline BDI 2011-100 & 2,44 & 3,00 \\
\hline BD-17 & 3,00 & 2,56 \\
\hline BDI 2011-86 & 2,33 & 3,00 \\
\hline PA 26 & 2,67 & 2,67 \\
\hline BDI 2011-55 & 2,89 & 2,56 \\
\hline BDI 2011-36 & 2,22 & 2,33 \\
\hline HSF 0506 & 1,00 & 1,33 \\
\hline PA 06 & 2,56 & 2,33 \\
\hline BR Roxa & 1,44 & 1,67 \\
\hline
\end{tabular}

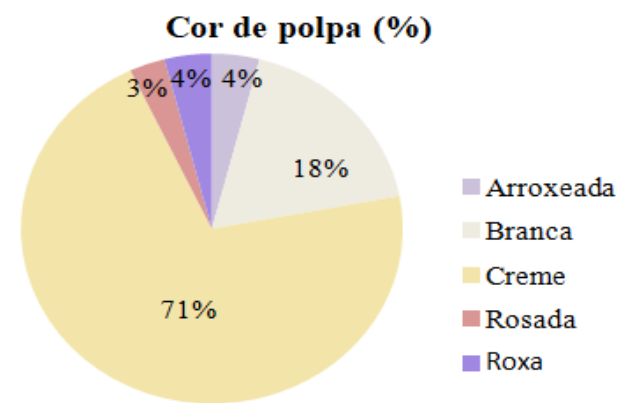

Cor de casca

Branca

Branca

Branca

Rosada

Branca

Branca

Roxa

Roxa

Roxa

Branca

Rosada

Branca

Branca

Roxa

Rosada

Branca

Branca

Branca

Branca

Branca

Branca

Branca

Rosada

Branca

Branca

Branca

Rosada
Cor de polpa

Creme

Creme

Creme

Branca

Creme

Creme

Creme

Creme

Rosada

Creme

Creme

Branca

Creme

Creme

Arroxeada

Branca

Creme

Branca

Creme

Creme

Branca

Creme

Roxa

Creme

Creme

Creme

Creme

Creme

BR Roxa: cultivar testemunha Brazlândia Roxa.

A cor predominante de casca foi branca, $(64,3 \%)$. A cor predominante de polpa foi a creme $(71,4 \%)$, semelhante ao encontrado por Veasey et. al. (2007) que observou para os genótipos do Vale do Ribeira, a cor predominante da polpa foi creme (73\%).

Vizzotto et al. (2018) avaliando a concentração de minerais em genótipos de batata-doce, de diferentes colorações de polpa, encontrou resultados que mostram que o teor de cálcio foi maior na em polpas de coloração creme.
As variedades de batata-doce mais comercializadas no Brasil são as que possuem polpa de coloração amarelada, branca e creme (MIRANDA et al, 1995; MELO et al., 2010).

Entre as duas cultivares que apresentaram melhores notas de formato e resistência a insetos, HSF 0506 (casca branca e polpa creme) e BDI 2011-52 (casca rosada e polpa arroxeada), em termos de cor de casca e polpa a primeira se torna mais atrativa ao consumo de mesa. 
É importante identificar potencial de mesa em genótipos melhorados para produção de etanol revelando inclusive dois mercados que podem absorver estes materiais, sendo um aspecto favorável para se trabalhar de acordo com a demanda do mercado.

\section{CONCLUSÃO}

Segundo os dados avaliados, conclui-se que a cultivar HSF 0506 se mostrou superior às outras, atendendo todos os padrões de mesa, tendo notas de formato e resistência a insetos semelhantes a BDI 2011-52, porém se destacando em comparação a BDI 2011-52, por possuir coloração da polpa cor de creme, o que é mais desejado pelos consumidores, segundo, bibliografias estudadas.

Todos os autores declararam não haver qualquer potencial conflito de interesses referente a este artigo.

\section{REFERÊNCIAS}

AZEVEDO, S.M.; FREITAS, J.A.; MALUF, W.R.; SILVEIRA, M.A. Desempenho de clones e métodos de plantio de batata-doce. Acta Scientiarum. v.22, n.4, p.901-905, 2000.

BEVILAQUA, L.K.S.; MOTA, J. H.; RESENDE, G. M. de; YURI, J. E. Características morfológicas e produtivas de clones de batata doce. Cad. Ciênc. Agrárias., v. 11, p. 01-07, 2019.

CAMARGO, L.K.P.; RESENDE, J.T.V.; MÓGOR, A.F.; CAMARGO, C.K. ; KURCHAIDT, S.M. Uso de índice de seleção na identificação de genótipos de batata doce com diferentes aptidões. Horticultura Brasileira. v. 34, n. 4, p. 514-519, 2016.

EMBRAPA. Batata-Doce Brazlândia Roxa. 2017. Disponível em: https://www.embrapa.br/busca-desolucoes-tecnologicas/-/produto-servico/601/batatadoce-brazlandia-roxa. Acesso em: 15/03/2019.

FURLANETO, F.P.R.; FIRETTI, R.; MONTES, S.M.N.M. Comercialização, custos e indicadores de rentabilidade da batata doce. Pesquisa $\&$ Tecnologia. vol. 9, n. 2, 2012.
KALKMANN, D. C. Produtividade, qualidade de raiz, resistência aos insetos de solo e aos nematóides-dasgalhas, e estimativas de parâmetros genéticos em clones de Batata-doce cultivados no Distrito Federal. Brasília-DF. (Dissertação de mestrado). Universidade de Brasília-UNB; 2011.

NEUMANN, E. R.; RESENDE, J.T.V.; CAMARGO, L.K.P.; CHAGAS, R.R.; FILHO, R.B.L. Produção de mudas de batata doce em ambiente protegido com aplicação de extrato de Ascophyllum nodosum. Horticultura Brasileira. v. 35, n. 4, p. 490498, 2017.

MELO, W.F.; SILVA, J.B.C.; MOITA, A.W. Avaliação da produtividade de clones de batata-doce ricos em provitamina A junto a agricultores familiares. Horticultura Brasileira, vol. 28, n 2, 2010.

MIRANDA, J.E.C. de; FRANCA, F.H.; CARRIJO, O. A.; SOUZA, A. F.; PEREIRA, W.; LOPES, C.A.; SILVA, J.B.C. A cultura da batata-doce. Embrapa-SPI: Embrapa-CNPH. Brasília, DF, 1995.

MIRASSE, J. J. O consumo de batata-doce de polpa alaranjada entre famílias rurais do nordeste de Moçambique: um estudo sobre percepções de comida e Segurança Alimentar na província de Nampula. Porto Alegre-RS (Dissertação de mestrado). Universidade Federal do Rio Grande do Sul-UFRGS; 2010.

SANTANA, W. R. Obtenção de genótipos de batatadoce (Ipomoea batatas (1.) lam.) por cruzamentos biparentais visando a produção de etanol. Palmas-TO. (Dissertação de mestrado). Universidade Federal do Tocantins- UFT. Palmas- TO; 2013.

SEPLAN-Secretaria do Planejamento e da Modernização da Gestão Pública. Atlas do Tocantins: subsídios ao planejamento da gestão territorial. 2012.

SILVA, J.B.C.; LOPES, C.A.; MAGALHÃES, J. S. Batata-doce (Ipomoea batatas). Embrapa hortaliças. Sistemas de produção. Versão eletrônica. 2008. Disponível em: https://sistemasdeproducao.cnptia.embrapa.br/Fontes HTML/Batata-doce/Batata-

doce_Ipomoea_batatas/clima_solo.html. Acesso em 20/01/2020.

SILVA, G.O.; VENDRAME, L.P.C.; AMARO, G.B. Desempenho de clones de batata-doce para caracteres de rendimento e qualidade de raiz. Embrapa Hortaliças-Boletim de Pesquisa e Desenvolvimento (INFOTECA-E), 2019. 
VIZZOTTO, M.; PEREIRA, E. S.; CASTRO, L.A.S.;

VEASEY, E.A.; SILVA, J.R.Q.; BORGES, A.; BRESSAN, E.A.; PERONI, N. Fenologia e diversidade morfológica de raças de batata-doce (Ipomoea batatas ) do Vale do Ribeira. Scientia Agricola. vol.64, n.4, p.416-427, 2007.
RAPHAELLI, C.O., KROLOW, A.C. Composição mineral em genótipos de batata-doce de polpas coloridas e adequação de consumo para grupos de risco. Brazilian Journal of Food Technology. v. 21, p. 2-8, 2018. 